\title{
Physics and decisions: an inverted perspective
}

\author{
Shabnam Mousavi ${ }^{1}$. Shyam Sunder ${ }^{2}$
}

Received: 21 February 2020 / Accepted: 25 May 2020 / Published online: 21 July 2020

(c) The Author(s) 2020

In social science, we seek to understand human decisions in terms of social-psychological primitives: a decision maker or conscious self, aware of its opportunity sets, resource constraints, preferences, and somewhat contentiously, endowed with at least a spoonful of free will. Tired of swimming and reluctant to brave cold rapids, one decides to devise a bridge of logs. Without the self-conscious and the associated free will, this concept of decision would need some as yet unknown redefinition.

Physics and biology face no such challenge. A rock rolling down the hillside does not decide to crush the car when their paths cross, and a peacock does not choose its beautiful feathers to attract a mate. A general optimizing principle-minimizing action subject to constraints-is sufficient to organize our understanding of such phenomena without resorting to self-consciousness, preferences, or free will.

Between the two approaches - the laws-of-nature in natural science and conscious decision-making in social science, we do not have to choose one or the other to model and understand human behavior. We suggest, instead, a syncretic method to gain an understanding of human behavior in three tiers, best exemplified in physics, biology, and social sciences respectively. This note outlines our proposal, explained in more details elsewhere (Mousavi and Sunder 2019).

\section{Physical corpora are subject to physical laws}

A physical corpus is a common attribute of inanimate objects, animals, and humans, all subject to universal physical laws. Both a person and a stone fall to earth under the same laws of gravity independent of whether wind, accident, mischief, or suicidal intent is behind the event. Further, consider three examples of movement from a starting point to a target through a change in medium along the way (Fig. 1): where

Shabnam Mousavi

mousavi@mpib-berlin.mpg.de

Shyam Sunder

shyam.sunder@yale.edu

1 Max Planck Institute for Human Development, Berlin, Germany

2 Yale University, New Haven, USA 

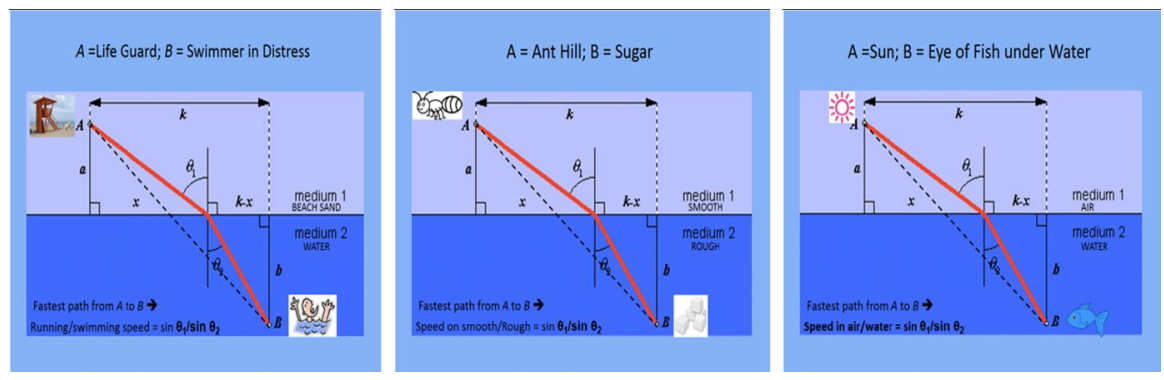

Fig. 1 A lifeguard, an ant, and a sunbeam: all follow a kinked path across two media

(a) a lifeguard runs over a sandy beach and then swims to reach a child; (b) an ant crawls between its nest located on a smooth surface and a food source located on a rough surface; and (c) a sunbeam travels from the sun to an eye of an underwater fish through vacuum and water. In all three cases, the fastest path (the kinked thick red line) is not the same as the shortest path (the broken straight line) connecting the starting point and target point.

In all these three different contexts, problems, and actors, a single general physics principle - the principle of least action-provides a simple solution. Published by Maupertuis in 1744, this principle generates a path-the kinked line in these three examples - along which the integral of the difference between the kinetic energy and potential energy, at every point in time, is minimized (Feynman 1963). We propose using this principle to isolate the elements of all forms of action-human, animal, or inanimate-that arises from the physical corpus involved; for inanimate, we need to proceed no further, and explanation of the remainder for animals can be sought in their biology, and for humans in their biology and higher social-psychological faculties.

Proposition Of all possible paths from a beginning point $A$ to an end point $B$, the materially efficient path uses minimal action, where action is a scalar that corresponds to the dimension in which (appropriately defined) value has been conserved.

\section{Inverting the order of deployment of rules}

The ability of the least action principle to capture inanimate dynamics so well does not imply that the least action path can either describe or serve as a normative standard for animal or human behavior. Instead, representation of an inanimate object's observed behavior in the form of such a path is only the first of three frames employed. Physics (the first frame) should hardly be complete for animate phenomenon. At the second level, laws and rules of biology are used to seek an understanding of what remains unexplained from the first (physical) frame. Beyond that, we use the third-level frame of social-psychological laws or regularities. The residual 
variation in observed phenomena after application of the three frames is attributable to the free will of humans (and at least some if not all animals).

Instead of debating if human and animal behavior is, or is not, mechanical, our framework simply incorporates the possibility that some of animal and human behavior is mechanical, and some of human behavior may be driven by biology instead of social psychology. This framework can be visualized as spherical Russian dolls analogous to earth with a physical core inside a biological mantle, wrapped in a social-psychological crust (Fig. 2). Extant approaches to theorizing and modeling human action start with social-psychological attributes - the crust, so to speak. We propose starting from the physical core and giving physics and biology a chance before resorting to the unique higher faculties of humans for explanations. Thus, we propose inverting the conventional approach.

\section{Using the principle of least action to frame animate and inanimate actions}

Table 1 illustrates six examples of the use of the principle of least action as an organizing principle for framing observed actions in terms of varied exogenous elements (Column 2), action element (Column 3), and the resulting path (Column 5). An action, here, is defined simply as a movement from a beginning point $A$ to an endpoint $B$.

Row 1 configures the simplest case of physical movement from point $A$ to point $B$, where both points are exogenously given on a plane, the action element is Euclidean distance, which is minimized on a straight line. Row 2 presents a familiar path of minimum action that a ball thrown in the air takes in the gravitational force field to return to the ground. Row 3 provides a decomposition of the phenomena in Fig. 1, captured in Snell-Descartes law, as an application of the general principle of least action here, time instead of distance (the fourth dimension in Minkowski space) (Minkowski 1908).

Row 4 implements the idea of using physics to frame observed behavior. The action element here is not a new dimension but a physical attribute, the (change

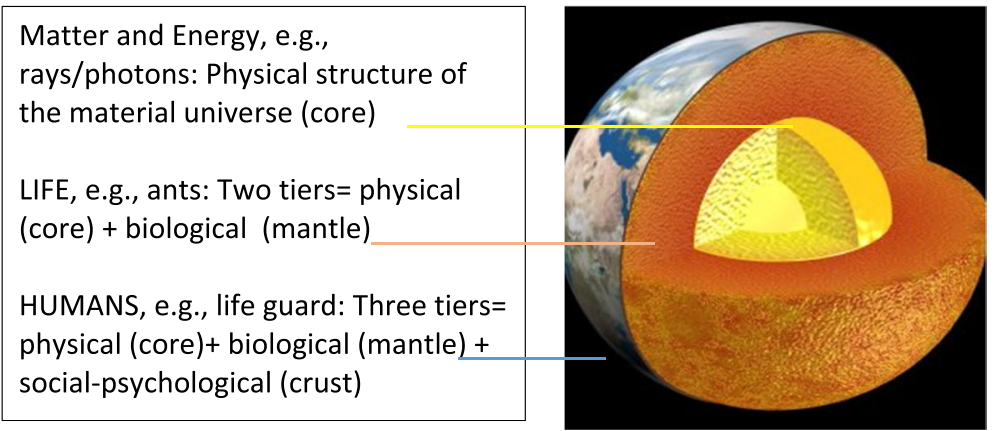

Fig. 2 Representing the tiers involved in modeling decisions by analogy 


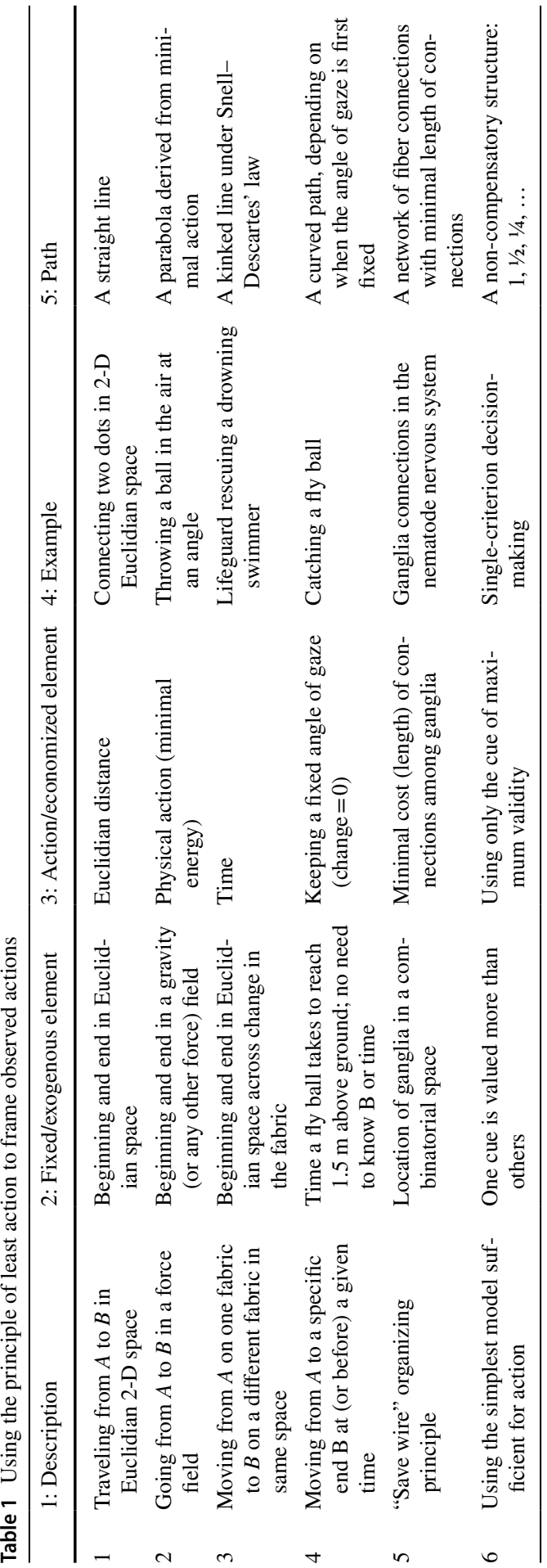


in the) angle of catcher's gaze. The exogenous element is time, since the execution of action does not require the actor's advance knowledge of the specific endpoint. We do not reject the possibility that a biological construct may be at work here (McBeath et al. 1995)—referred to in the literature as the gaze heuristic (Hamlin 2017). However, framing in physics requires only keeping the changes to a minimum (ideally zero), which is based not on the evolutionary capacity of maintaining the gaze (that resides in the biological brain) but on a physical element. Thus, this configuration remains in the physical core, without drawing on biological capabilities.

Row 5 takes a biological phenomenon-connections among the ganglia of the neural system in a tiny worm (nematode) — that minimizes the total length of wiring. This configuration assumes fixed ganglia locations for which connecting paths have been optimized, and thus does not include the possibility that the location of ganglia and connections are co-determined (Cherniak 1994). With this caveat, external elements are specified along an efficient path (network of connections) resulting from a minimization of action as measured by the length of connections.

Row 6 represents problem-solving behavior as moving from the problematic beginning $A$ to a resolved end $B$. Here, the actor is devising a simple heuristic, single-criterion decision-making, for solving the problem at hand (Gigerenzer and Gaissmaier 2011; Mousavi et al. 2017). This action can be framed in physics as follows: the external requirements or non-action elements are specific structures in the task environment that lend themselves to a solution that results from considering the single cue with the highest validity and ignoring the rest. Here, economizing an action element does not involve the cognitive effort spent on the search for relevant cues and the subsequent choice of only one cue/reason from the set of all available cues. We frame the action only after such cue has been chosen. The path in this case can be configured as an abstract interpretation, a mathematical series that corresponds to the non-compensatory structure of the cues' environment (Kvam and Pleskac 2017).

Our agenda consists of populating this table-i.e., representing observed behavior in terms of an action element, exogenous factors, and a path-to generate physical configurations of observed behaviors in terms of the principle of least action. As we move forward with our broader agenda, it is plausible to expect that other physical laws will gradually enter the stage.

In summary, we propose giving laws from classical physics the first chance for framing the actions observed not only in material, but also in animate worlds. Matter and energy included in biological domains do not lose their physicality by virtue of the added DNA, brain, self-consciousness, higher faculties, and social context in which individuals grow up and live. The benefit of starting at the physical core is to eliminate the necessity to justify the relevance of this work to human behavior for modelers who inevitably use physical frames. Ours is not a reductionist proposal to claim that everything can be explained by physics or by anything else. Each of the three tiers in our framework has a place of its own within which its concepts, rules and regularities are best applied. 
Acknowledgments Open access funding provided by Projekt DEAL.

Open Access This article is licensed under a Creative Commons Attribution 4.0 International License, which permits use, sharing, adaptation, distribution and reproduction in any medium or format, as long as you give appropriate credit to the original author(s) and the source, provide a link to the Creative Commons licence, and indicate if changes were made. The images or other third party material in this article are included in the article's Creative Commons licence, unless indicated otherwise in a credit line to the material. If material is not included in the article's Creative Commons licence and your intended use is not permitted by statutory regulation or exceeds the permitted use, you will need to obtain permission directly from the copyright holder. To view a copy of this licence, visit http://creativecommons.org/ licenses/by/4.0/.

\section{References}

Cherniak C (1994) Component placement optimization in the brain. J Neurosci 14(4):2418-2427

Feynman R (1963) The Feynman lectures on physics. http://www.feynmanlectures.caltech.edu/info/ Gigerenzer G, Gaissmaier W (2011) Heuristic decision making. Ann Rev Psychol 62:451-482

Hamlin RP (2017) The gaze heuristic: biography of an adaptively rational decision process. Top Cogn Sci 9:264-288

Kvam PD, Pleskac TJ (2017) A quantum information architecture for cue-based heuristics. Decision 4(4):197-233

McBeath MK, Shaffer DM, Kaiser MK (1995) How baseball outfielders determine where to run to catch fly balls. Science 268:569-573

Minkowski H (1908) Raum und Zeit. [Space and time] Physikalische Zeitschrift 10:75-88. English translation available at: www.minkowskiinstitute.org

Mousavi S, Sunder S (2019) Physical laws and human behavior: a three-tier framework. Cowles foundation discussion papers, no. 2173. https://doi.org/10.31219/osf.io/nmpc8

Mousavi S, Gigerenzer G, Kheirandish R (2017) Rethinking behavioral economics through fast-and-frugal heuristics. In: Frantz R, Chen S, Dopfer K, Mousavi S (eds) Routledge handbook of behavioral economics. Routledge, Abingdon, pp 280-296

Publisher's Note Springer Nature remains neutral with regard to jurisdictional claims in published maps and institutional affiliations. 